\title{
Comparison of surgical techniques for stoma closure: A retrospective study of purse-string skin closure versus conventional skin closure following ileostomy and colostomy reversal
}

\author{
YUMA WADA, NORIKATSU MIYOSHI, MASAYUKI OHUE, SHINGO NOURA, SHIKI FUJINO, \\ KEIJIROU SUGIMURA, HIROFUMI AKITA, MASAAKI MOTOORI, KUNIHITO GOTOH, \\ HIDENORI TAKAHASHI, SHOGO KOBAYASHI, TAKESHI OHMORI, \\ YOSHIYUKI FUJIWARA and MASAHIKO YANO
}

Department of Surgery, Osaka Medical Center for Cancer and Cardiovascular Diseases, Osaka 537-8511, Japan

Received November 10, 2014; Accepted January 8, 2015

DOI: $10.3892 / \mathrm{mco} .2015 .505$

\begin{abstract}
The aim of this study was to compare the incidence of postoperative complications, including superficial incisional surgical site infection (SSI) following purse-string skin closure (PS) and conventional skin closure with a drainage tube (CD) following stoma closure. A total of 55 consecutive patients who underwent loop colostomy and loop ileostomy closures in our hospital between October, 2011 and September, 2014 were retrospectively assessed. The patients were divided into two groups, namely the PS group (26 patients) and the CD group (29 patients). There were no significant differences in the characteristics of the patients between the two groups. The baseline and operative characteristics also did not differ significantly between the two groups. However, the incidence of superficial incisional SSI was lower in the PS group compared to that in the CD group ( 0 vs. $13.8 \%$, respectively; $\mathrm{P}=0.049$ ). The overall incidence of complications did not differ significantly between the two groups $(\mathrm{P}=0.313)$. The duration of postoperative hospital stay in the PS group was shorter compared to that in the CD group. In conclusion, the results of this study suggest that PS may an effective technique to reduce the incidence of superficial incisional SSI. This technique appears to be superior to the conventional technique, allowing for better cosmesis.
\end{abstract}

Correspondence to: Dr Norikatsu Miyoshi, Department of Surgery, Osaka Medical Center for Cancer and Cardiovascular Diseases, 1-3-3 Nakamichi, Osaka 537-8511, Japan

E-mail: miyosi-no@mc.pref.osaka.jp

Key words: stoma closure, purse-string skin closure, surgical site infection, drainage

\section{Introduction}

Stoma closure is considered a minimally invasive surgery; however, surgical site infection (SSI) is a frequent complication. Recently, the number of cases of diverting ileostomy has increased due to the increase in the number of anal-preserving surgeries, such as super-low anterior resection (sLAR) and intersphincteric resection (ISR) for rectal cancer. In this study, we focused on methods used to decrease the incidence of SSI in stoma closure.

The incidence of wound infection following stoma closure ranges between 2 and $41 \%$ across different studies $(1,2)$. The most frequent cause of wound infection is bacterial contamination of the skin surrounding the ileostomy/colostomy due to prolonged contact with bowel contents or due to leakage of the ileostomy contents. A previous study in 1997 introduced a purse-string skin closure technique (PS) for ileostomy reversal, which reduced the risk of wound infection and resulted in smaller scars, allowing for better cosmesis (3).

The number of studies investigating the effectiveness of PS is currently limited. We previously used a Penrose drainage tube under the subcutaneous tissue to prevent SSI and have reported the efficacy of this technique in our hospital (4).

PS was reported to be better for controlling superficial incisional SSI $(5,6)$ and we introduced this technique in June, 2013. In the present study, we examined surgical techniques by either conventional skin closure with a drainage tube (CD) or PS and compared the incidence of superficial incisional SSI between CD and PS in order to assess the efficacy of the PS in ileostomy and colostomy reversal.

\section{Patients and methods}

Patients. We retrospectively reviewed the medical records of 55 consecutive patients who underwent stoma closure. A total of 26 patients who underwent PS between June, 2013 and September, 2014 were compared to 29 patients who underwent CD between October, 2011 and May, 2013 at the Osaka Medical 
Table I. Centers for Disease Control and Prevention guidelines for the diagnosis of superficial SSI.

Characteristics of superficial SSI

1. Purulent drainage, with or without laboratory confirmation, from the superficial incision.

2. Organisms isolated from an aseptically-obtained culture of fluid or tissue from the superficial incision.

3. At least one of the following signs or symptoms of infection: Pain or tenderness, localized swelling, redness or heat and superficial incision deliberately opened by surgeon, unless the incision is culture-negative.

4. Diagnosis of superficial incisional SSI by the surgeon or attending physician.

Superficial SSI occurs within 30 days of an operation and involves only the skin or subcutaneous tissue surrounding the incision and at least one of the abovementioned characteristics. SSI, surgical site infection.
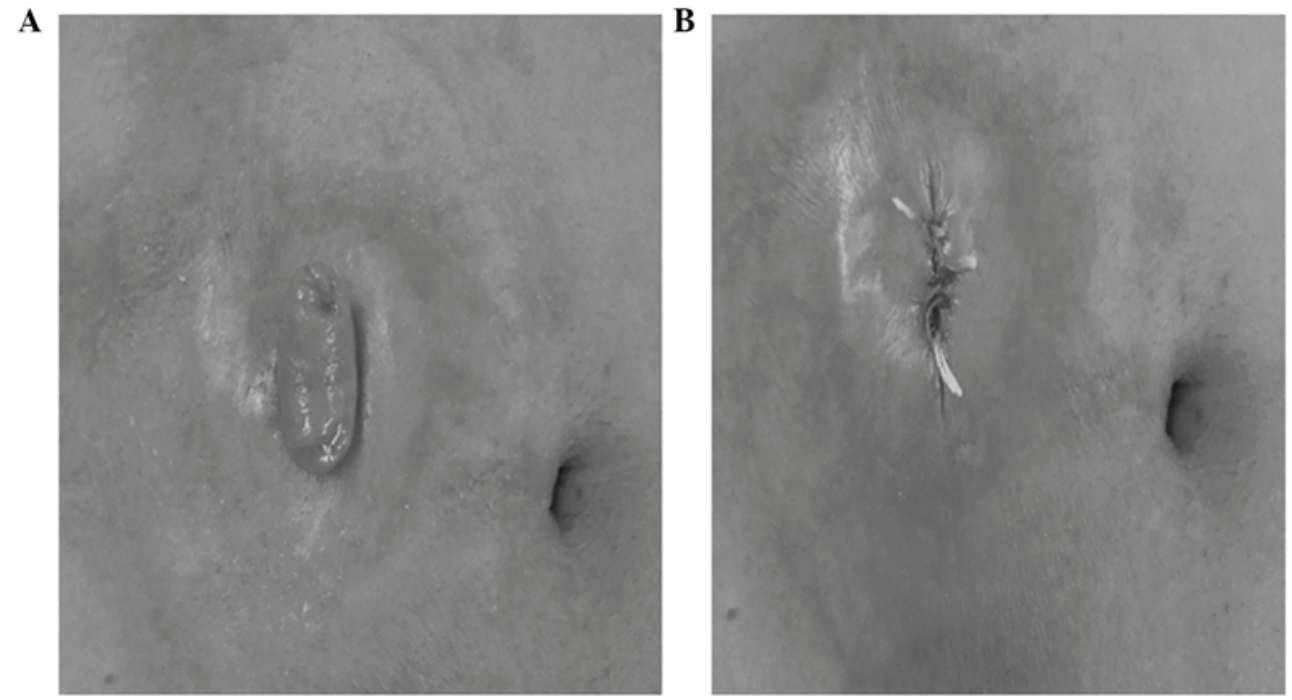

Figure 1. Surgical procedure of the purse-string skin closure. (A and B) Following irrigation with saline, an open-ileostomy/colostomy was closed by sutures. The skin was cut around the sutured stoma while maintaining a $\sim 3-5 \mathrm{~mm}$ margin.

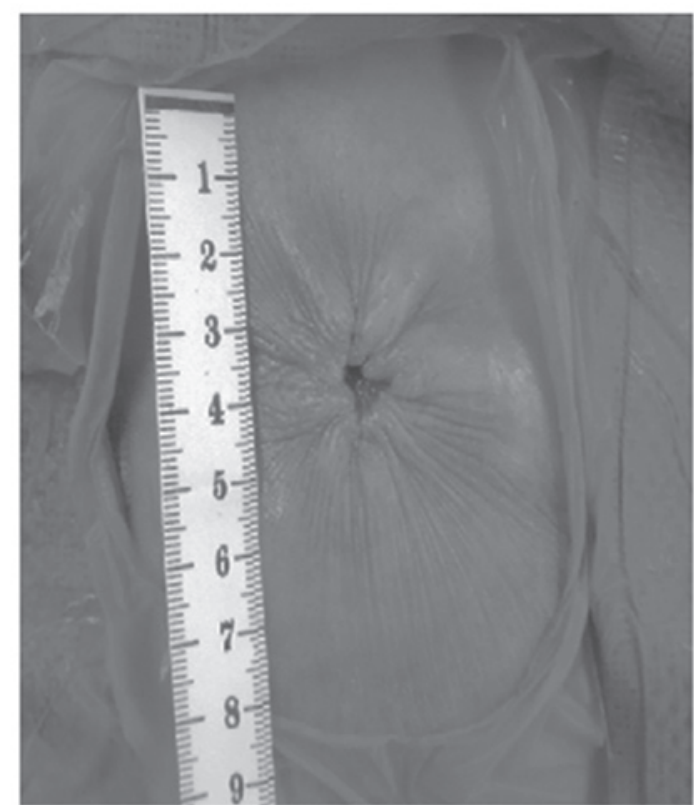

Figure 2. Postoperative picture of the purse-string skin closure. Approximately $5 \mathrm{~mm}$ of the center of the wound were mainained open to drain the discharge from the subcutaneous wound.
Center for Cancer and Cardiovascular Diseases, Japan. Medical charts were reviewed for patient demographics, including age, gender and body mass index; past medical history, such as the presence of diabetes, chronic obstructive pulmonary disease, cardiovascular disease and liver dysfunction; alcohol consumption (regular vs. moderate or non-drinker), smoking status (smoked within 1 year prior to the operation), medication records, including perioperative steroid replacement; American Society of Anesthesiology (ASA) score; and preoperative serum albumin values for the CD and PS groups.

This study was approved by the Institutional Review Board of Osaka Medical Center for Cancer and Cardiovascular Diseases and written informed consent was obtained from all the patients.

Diagnosis of SSI. Wound infection was defined as the presence of cellulitis or purulent discharge, with or without positive bacterial growth, within 30 days after the operation (adopted from the 1992 Centers for Disease Control and Prevention definition of SSI) (Table I). The surgical wounds were routinely observed and evaluated by the surgical team and surveyed until 30 days after the operation. 
Table II. Patient characteristics $(\mathrm{n}=55)$.

\begin{tabular}{|c|c|c|c|}
\hline Characteristics & $\mathrm{CD}(\mathrm{n}=29)$ & PS $(n=26)$ & P-value \\
\hline Gender & & & 0.738 \\
\hline Male & 20 & 19 & \\
\hline Female & 9 & 7 & \\
\hline Age, years & $58(34-79)$ & $65(27-80)$ & 0.255 \\
\hline Body mass index & $22(16-27)$ & $22(14-29)$ & 0.831 \\
\hline ASA score & & & NA \\
\hline 1 & 9 & 9 & \\
\hline 2 & 20 & 14 & \\
\hline 3 & 0 & 3 & \\
\hline $\begin{array}{l}\text { Preoperative albumin } \\
\text { serum value }\end{array}$ & $4.1(3.4-5.7)$ & $4.1(3.7-4.9)$ & 0.799 \\
\hline \multicolumn{4}{|l|}{$\begin{array}{l}\text { Preoperative } \\
\text { comorbidities }\end{array}$} \\
\hline Diabetes & 1 & 0 & NA \\
\hline Cardiovascular disease & 2 & 3 & NA \\
\hline COPD & 1 & 0 & NA \\
\hline Steroid use & 0 & 0 & NA \\
\hline Alcohol consumption & 7 & 10 & 0.251 \\
\hline Smoking & 7 & 6 & 0.926 \\
\hline Liver cirrhosis & 0 & 0 & NA \\
\hline Ileostomy/colostomy & $26 / 3$ & $22 / 4$ & NA \\
\hline Anastomosis (FEEA/AL) & $27 / 2$ & $24 / 2$ & NA \\
\hline
\end{tabular}

All the continuous variables are expressed as median (range). $\mathrm{CD}$, conventional skin closure with a drainage tube; PS, purse-string skin closure; ASA, American Society of Anesthesiology; COPD, chronic obstructive pulmonary disease; FEEA, functional end-to-end anastomosis; AL, Albert-Lembert anastomosis; NA, not available.

Surgical technique. All the patients underwent preoperative mechanical bowel preparation. After the induction of general anesthesia, prophylactic antibiosis (cefmetazole, $2 \mathrm{~g} /$ day) was administered. An open-ileostomy/colostomy was sutured (Fig. 1A and B) and the wound was irrigated by povidone iodine and $500 \mathrm{ml}$ of saline. The skin around the sutured stoma was resected, maintaining a $\sim 3-5 \mathrm{~mm}$ margin. The intestinal tracts leading to the stoma were extracted from the abdominal cavity and dissection of the sutured stoma was performed, followed by a functional end-to-end anastomosis or Albert-Lembert anastomosis. The peritoneum and rectus fascia were closed using Vicryl 1.0; (Johnson \& Johnson Co., New Brunswick, NJ, USA). Antibiotics were administered for 3 days postoperatively.

In the CD group, the skin site was closed primarily with a skin stapler. At the stoma site, a Penrose drainage tube was left subcutaneously. In the PS group, the dermis layer was sutured, drawing a purse-string form using polydioxanone 3.0 suture (Johnson \& Johnson Co.). It is recommended that $\sim 5 \mathrm{~mm}$ of the center of the wound are maintained open to drain any discharge (Fig. 2).

Statistical analysis. The data presented were analyzed using the Pearson's Chi-square and Fisher's exact tests. For
Table III. Perioperative factors in conventional skin closure with a drainage tube (CD) and purse-string skin closure (PS).

\begin{tabular}{|c|c|c|c|}
\hline Factors & CD (n=29) & PS $(n=26)$ & $\mathrm{P}$-value \\
\hline Operative time, $\min$ & $98(53-213)$ & $106(60-190)$ & 0.377 \\
\hline Blood loss, ml & $15(0-130)$ & $20(0-130)$ & 0.203 \\
\hline Wound length, $\mathrm{cm}$ & $6(4-8)$ & $0.5(0.5-1)$ & $<0.001$ \\
\hline Complications & 5 & 2 & 0.313 \\
\hline Wound infection & 4 & 0 & 0.049 \\
\hline $\begin{array}{l}\text { Small bowel } \\
\text { obstruction }^{\mathrm{a}}\end{array}$ & 2 & 1 & NA \\
\hline $\begin{array}{l}\text { Anastomosis } \\
\text { leakage }^{\mathrm{a}}\end{array}$ & 0 & 0 & NA \\
\hline $\begin{array}{l}\text { Postoperative } \\
\text { bleeding }^{\mathrm{a}}\end{array}$ & 0 & 1 & NA \\
\hline $\begin{array}{l}\text { Postoperative } \\
\text { hospital stay, days }\end{array}$ & $14(9-50)$ & $13(8-29)$ & 0.159 \\
\hline
\end{tabular}

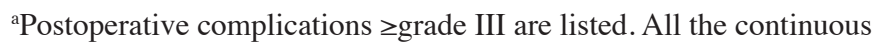
variables are expressed as median (range). NA, not available.

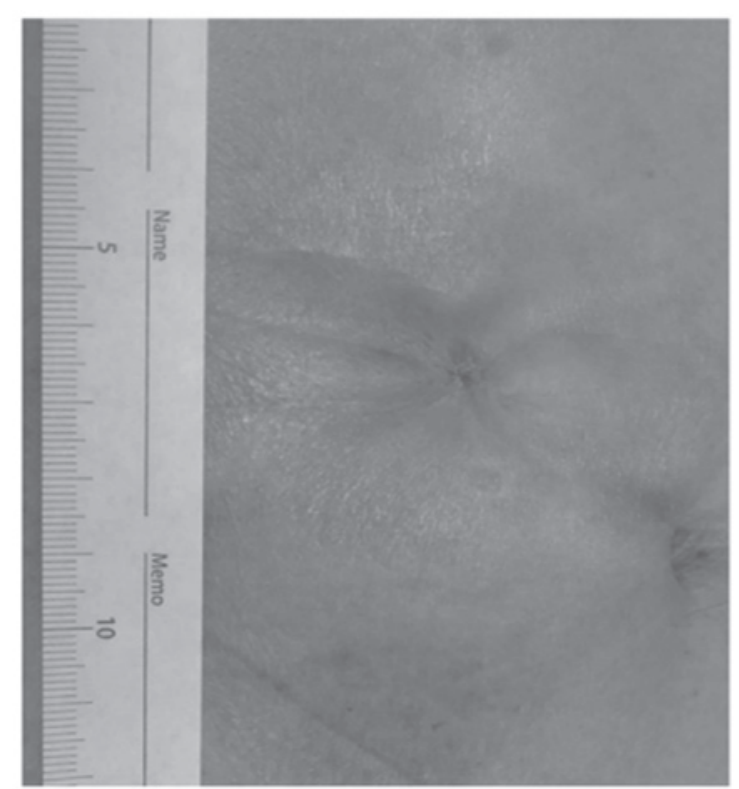

Figure 3. Postoperative picture 1 month following purse-string skin closure. The scar became smaller, allowing for better cosmesis.

continuous variables, data were expressed as median (range). The Mann-Whitney U-test was used for statistical comparison of different groups. $\mathrm{P}<0.05$ was considered to indicate a statistically significant difference. All the tests were analyzed using JMP software, version 11.0 (SAS Institute, Cary, NC, USA).

\section{Results}

Patient characteristics. The patient characteristics are summarized in Table II. There were no significant differences in patient characteristics, such as age, gender, body mass index, 
preoperative comorbidities, ASA scores and preoperative serum albumin values between the CD and PS groups. The perioperative factors of the $\mathrm{CD}$ and $\mathrm{PS}$ groups are summarized in Table III. The median operative time of the CD group was $98 \mathrm{~min}$ (range, 53-213 min) and that of the PS group $106 \mathrm{~min}$ (range, 60-190 $\mathrm{min}$ ); this difference was not statistically significant $(\mathrm{P}=0.377)$. In the $\mathrm{CD}$ group, the median blood loss was $15 \mathrm{ml}$ (range, $0-130 \mathrm{ml}$ ), whereas in the PS group, it was $20 \mathrm{ml}$ (range, $0-130 \mathrm{ml})(\mathrm{P}=0.203)$. The median wound length in the PS group was $0.5 \mathrm{~cm}$, which was significantly shorter compared to that in the $\mathrm{CD}$ group $(6 \mathrm{~cm})(\mathrm{P}<0.001)$. As regards postoperative complications, superficial incisional SSI in the CD group was observed in 4 patients (13.8\%); however, in the PS group, SSI was not observed. Thus, there was a significant difference in the incidence of superficial incisional SSI between the CD and PS groups $(\mathrm{P}=0.049)$. With regard to other complications, small bowel obstruction was observed in 2 patients (6.9\%) in the $\mathrm{CD}$ group and 1 patient (3.8\%) in the PS group. In the $\mathrm{CD}$ group, postoperative bleeding was observed in 1 patient (3.8\%). Anastomosis leakage was not observed in any of the two groups. The overall incidence of complications in the CD and PS groups was not significantly different $(\mathrm{P}=0.313)$. The median postoperative hospital stay was 14 days (range, 9-50 days) in the $\mathrm{CD}$ group and 13 days (range, 8-29 days) in the PS group.

\section{Discussion}

Wound infection (particularly, superficial incisional SSI) is the most common complication following stoma closure, reportedly occurring in one of every three cases $(7,8)$. A prospective review of the complications of ileostomy closure reported an SSI rate of $18.3 \%$ (9). The high incidence rate of SSI was considered to be associated with the primary closure of these wounds. Generally, superficial incisional SSI is not a fatal complication; however, there is a risk of severe infection and abdominal wall scar hernia. Therefore, we reported the utility of the Penrose drain placement under the subcutaneous tissue for reducing the incidence of SSI (4). However, with this technique, wound infection may not be sufficiently controlled; thus, we introduced PS for stoma closure in order to reduce the incidence of SSI.

In the present study, the SSI rate in the CD group was $8.3 \%$, which is consistent with previous reports $(10,11)$. However, no patients developed SSI in the PS group. This finding is in accordance with a previous report (12). PS of stoma reversal is a cosmetically superior approach (3). A recent study compared patients treated using PS to those treated using primary closure (12) and found that no SSI occurred in the PS group compared to the CD group, in which $40 \%$ of patients developed SSI $(\mathrm{P}=0.001)$. These results suggest a lower risk of SSI when PS is used. To effectively prevent SSI, it is important to maintain the drainage hole until the discharge from the wound is reduced (Fig. 2).

Recently, PS has been a focus of investigation with regard to better cosmetic appearance (Fig. 3) $(13,14)$. In the present study, the wound length was evaluated in both groups. PS was performed and $\sim 0.5 \mathrm{~cm}$ of the wound was maintained open in order to drain the discharge. By contrast, the patients in the $\mathrm{CD}$ group required a $\sim 6-\mathrm{cm}$ linear suture. Taken together, the results suggest that PS allows for better cosmesis in addition to the lower risk of SSI development.

With the improvement of surgical techniques for anal preservation by sLAR and ISR in patients with rectal cancer, the opportunities for primary artificial anus construction have increased. Stoma closure is associated with a high risk of wound infection; therefore, it is important to evaluate the usefulness of PS and open drainage system to prevent severe SSI. We are currently conducting a randomized trial to confirm our findings and assess additional end points, including cost-effectiveness and patient satisfaction.

In conclusion, the present study suggests that PS is associated with a lower risk of postoperative SSI compared to primary skin closure, even with the placement of a drainage tube; it also allows for better cosmesis, including a more favorable wound length, indicating that PS may be a favorable alternative to primary skin closure.

\section{References}

1. Hackam DJ and Rotstein OD: Stoma closure and wound infection: an evaluation of risk factors. Can J Surg 38: 144-148, 1995.

2. Wong KS, Remzi FH, Gorgun E, et al: Loop ileostomy closure after restorative proctocolectomy: outcome in 1,504 patients. Dis Colon Rectum 48: 243-250, 2005.

3. Banerjee A: Pursestring skin closure after stoma reversal. Dis Colon Rectum 40: 993-994, 1997.

4. Imada S, Noura S, Ohue M, et al: Efficacy of subcutaneous penrose drains for surgical site infections in colorectal surgery. World J Gastrointest Surg 5: 110-114, 2013.

5. Mirbagheri N, Dark J and Skinner S: Factors predicting stomal wound closure infection rates. Tech Coloproctol 17: 215-220, 2013.

6. Chow A, Tilney HS, Paraskeva P, Jeyarajah S, Zacharakis E and Purkayastha S: The morbidity surrounding reversal of defunctioning ileostomies: a systematic review of 48 studies including 6,107 cases. Int J Colorectal Dis 24: 711-723, 2009.

7. Vermulst N, Vermeulen J, Hazebroek EJ, Coene PP and van der Harst E: Primary closure of the skin after stoma closure. Management of wound infections is easy without (long-term) complications. Dig Surg 23: 255-258, 2006.

8. Williams LA, Sagar PM, Finan PJ and Burke D: The outcome of loop ileostomy closure: a prospective study. Colorectal Dis 10: 460-464, 2008

9. Garcia-Botello SA, Garcia-Armengol J, Garcia-Granero E, et al: A prospective audit of the complications of loop ileostomy construction and takedown. Dig Surg 21: 440-446, 2004.

10. Parks SE and Hastings PR: Complications of colostomy closure. Am J Surg 149: 672-675, 1985.

11. Pittman DM and Smith LE: Complications of colostomy closure. Dis Colon Rectum 28: 836-843, 1985.

12. Milanchi S, Nasseri Y, Kidner T and Fleshner P: Wound infection after ileostomy closure can be eliminated by circumferential subcuticular wound approximation. Dis Colon Rectum 52: 469-474, 2009.

13. Marquez TT, Christoforidis D, Abraham A, Madoff RD and Rothenberger DA: Wound infection following stoma takedown: primary skin closure versus subcuticular purse-string suture. World J Surg 34: 2877-2882, 2010.

14. Lee JR, Kim YW, Sung JJ, et al: Conventional linear versus purse-string skin closure after loop ileostomy reversal: comparison of wound infection rates and operative outcomes. J Korean Soc Coloproctol 27: 58-63, 2011. 